Case Report

\title{
Long-term stability of surgical-orthodontic treatment for skeletal Class III malocclusion with mild asymmetry
}

\author{
Seden Akan1), Ilken Kocaderel12), and Gokhan Tuncbilek ${ }^{3}$ ) \\ 1)Department of Orthodontics, School of Dentistry, Istanbul Medipol University, Istanbul, Turkey \\ 2)Department of Orthodontics, School of Dentistry, Hacettepe University, Ankara, Turkey \\ 3)Department of Plastic Reconstructive and Esthetic Surgery, School of Medicine, Hacettepe University, \\ Ankara, Turkey
}

(Received January 8, 2016; Accepted August 7, 2016)

\begin{abstract}
Skeletal Class III malocclusion with mandibular prognathism is often associated with mandibular asymmetry, and patients with CI III deformity and asymmetry may undergo orthognathic surgery to improve facial function and esthetics. However, the long-term stability of mandibular setback surgery has been considered "problematic". We report a 25-year-old female patient who was diagnosed as having a skeletal Class III relationship, mandibular asymmetry and unilateral crossbite. She underwent bilateral sagittal split-ramus osteotomy and orthodontic therapy, and this led to improvement of her dentofacial relationship, esthetics and occlusion. The outcome was stable after 8 years of retention. This case shows that asymmetry correction using mandibular setback surgery for skeletal Class III deformity can yield long-term stability.
\end{abstract}

Keywords: BSSO; skeletal Class III malocclusion; retention.

\section{Introduction}

Skeletal Class III malocclusion is one of the most complicated of all dentofacial abnormalities in both childhood

Correspondence to Dr. Seden Akan, Department of Orthodontics, School of Dentistry, Istanbul Medipol University, Ataturk Bulv. No: 27, Unkapani Fatih 34083, Istanbul, Turkey

E-mail: sedenakandt@hotmail.com

Color figures can be viewed in the online issue at J-STAGE. doi.org/10.2334/josnusd.16-0004

DN/JST.JSTAGE/josnusd/16-0004 and adulthood (1). It is characterized by mandibular prognathism and maxillary deficiency (although most patients with Class III malocclusion have combinations of both) and has a significant genetic component. Growth modification with a chin cup or face mask can be used for treatment of Class III malocclusion in growing patients, but in adult patients, if the severity of the deformity exceeds the dentoalveolar camouflage ranges, combined orthodontic and surgical treatment can help to achieve proper functional and esthetic rehabilitation.

Skeletal Class III malocclusion with mandibular prognathism is often associated with mandibular asymmetry, since the mandibular closure path is altered to achieve dental intercuspation, despite the sagittal deformity (2). Proffit et al. (3) have stated that patients' requests for orthognathic surgery tend not to be related to morphologic characteristics but rather to psychological factors, such as low self-esteem, self-dislike and psychological distress. Nicodemo et al. (4) reported that self-esteem after orthognathic surgery for Class III malocclusion was especially improved in female patients. However, the positive changes obtained through orthognathic surgery may be lost if postsurgical relapse occurs, leading to unsatisfactory treatment outcomes. Relapse rates after mandibular setback surgery are among the highest for any form of surgical procedure (5). Here we report a case of skeletal Class III malocclusion with mild mandibular asymmetry and unilateral crossbite for which a combination of surgery and orthodontic therapy achieved long-term stability. 


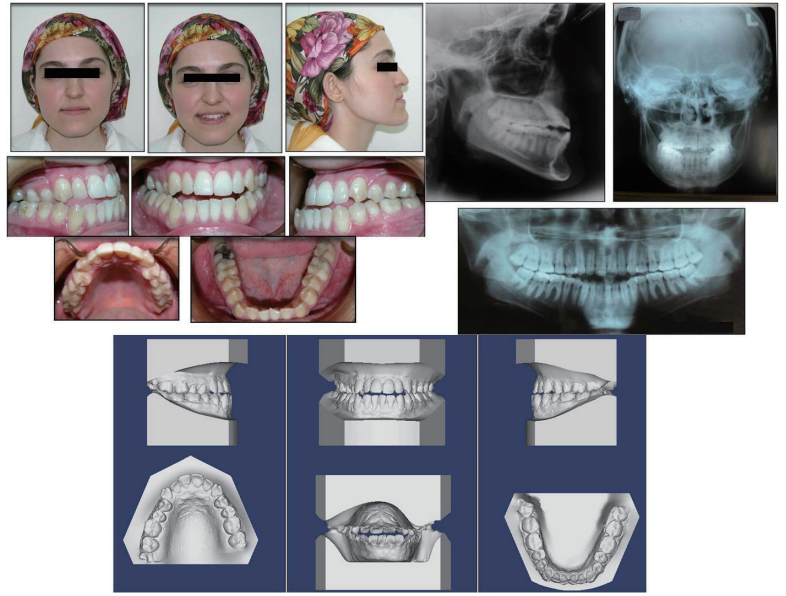

Fig. 1 Pretreatment facial and intraoral photographs, dental casts, and lateral cephalometric, posteroanterior and panoramic radiographs.

\section{Case Report}

A 25-year, 5-month-old female patient presented with complaints of mandibular protrusion and facial asymmetry (Fig. 1). Intraoral examination revealed a Class III molar and canine relationship on both sides: The mandibular midline was deviated $3 \mathrm{~mm}$ toward the right relative to the maxilla and facial midline, and there was both anterior and right posterior crossbite (Fig. 1). The overjet and overbite were -2.5 and $-2 \mathrm{~mm}$, respectively. The patient, who had no breathing disorder, obstructive sleep apnea, or relevant family or medical history, was motivated to receive treatment. A panoramic radiograph showed that all of her third molars had erupted completely (Fig. 1). Lateral and posteroanterior cephalograms were taken in the same head position using cephalostat at the T1 (pretreatment), T2 (post-treatment) and T3 (post-retention) stages. The cephalometric analysis demonstrated a skeletal Class III jaw base relationship, an average mandibular plane angle, increased inclination of the maxillary incisors, and normal inclination of the mandibular incisors (Table 1). The posteroanterior cephalogram showed a mandibular deviation of $3 \mathrm{~mm}$ toward the right (Table 1).

Treatment began in the maxillary and mandibular arches with Roth-type $(0.018 \times 0.025$ slot $)$ pre-adjusted brackets. A sequence of 0.014- and 0.016-inch nickeltitanium arches was used for leveling and alignment in the maxilla and mandible. Thereafter, tooth alignment was continued by changing the archwires sequentially; intercuspidation was checked by occluding the plaster
Table 1 Summary of the lateral and posteroanterior cephalometric analysis

\begin{tabular}{|c|c|c|c|}
\hline Measurement & \multicolumn{3}{|c|}{ Pretreatment Post-treatmentPost-retention } \\
\hline \multicolumn{4}{|l|}{ Skeletal pattern } \\
\hline SNA $\left({ }^{\circ}\right)$ & 79 & 79 & 79 \\
\hline $\mathrm{SNB}\left({ }^{\circ}\right)$ & 85 & 80 & 80 \\
\hline ANB $\left(^{\circ}\right)$ & -6 & -1 & -1 \\
\hline Wits appraisal (mm) & -4 & 0 & 0 \\
\hline GoGn-SN $\left(^{\circ}\right)$ & 32 & 34 & 34 \\
\hline Lower anterior face height $\left({ }^{\circ}\right)$ & 50 & 52 & 52 \\
\hline Gonial angle $\left(^{\circ}\right)$ & 133 & 135 & 136 \\
\hline \multicolumn{4}{|l|}{ Dental pattern } \\
\hline Upper incisor to $\mathrm{SN}$ plane $\left(^{\circ}\right)$ & 119 & 111 & 109 \\
\hline $\operatorname{IMPA}\left({ }^{\circ}\right)$ & 89 & 93 & 90 \\
\hline \multicolumn{4}{|l|}{ Profile } \\
\hline Lower lip to Ricketts E plane (mm) & -6 & -5 & -5 \\
\hline Upper lip to Ricketts E plane (mm) & -9 & -2 & -3 \\
\hline \multicolumn{4}{|l|}{ Tongue space and hyoid bone position } \\
\hline $\mathrm{D} 1(\mathrm{~mm})$ & 6 & 4 & 3 \\
\hline $\mathrm{D} 2(\mathrm{~mm})$ & 18 & 16 & 13 \\
\hline $\mathrm{C} 3 \mathrm{H}(\mathrm{mm})$ & 36 & 39 & 35 \\
\hline $\mathrm{SH}(\mathrm{mm})$ & 115 & 114 & 115 \\
\hline $\mathrm{NSH}\left({ }^{\circ}\right)$ & 90 & 88 & 91 \\
\hline \multicolumn{4}{|l|}{ Posteroanterior measurements } \\
\hline $\mathrm{AG}-\mathrm{CsPl}(\mathrm{mm})$ & 45 & 41 & 41 \\
\hline GA-CsPl (mm) & 40 & 40 & 40 \\
\hline ZR-AG (mm) & 60 & 59 & 59 \\
\hline ZL-GA (mm) & 59 & 59 & 59 \\
\hline
\end{tabular}

models, and when satisfactory intercuspidation was obtained, $0.017 \times 0.025$-inch stainless steel archwires were used for alignment stabilization. After presurgical orthodontic treatment for 1 year and 5 months, crimpable surgical hooks were applied to the arches, and bilateral sagittal split-ramus osteotomy (BSSO) was performed. The mandible was set back $2.5 \mathrm{~mm}$ on the right side, 5.5 $\mathrm{mm}$ on the left side and rotated $3 \mathrm{~mm}$ counterclockwise. Better tooth interdigitation and occlusion finalization were obtained after orthognathic surgery by using vertical intermaxillary elastics. Postsurgical orthodontic treatment was continued for 6 months. The overall treatment duration was 1 years and 11 months. Hawley retainers were applied for retention.

At the end of treatment, the anterior and posterior crossbites were corrected. Additionally, a Class I molar and canine relationship and ideal overjet, overbite and favorable intercuspidation were obtained. The mandibular midline was coincident with the maxillary and facial midline (Fig. 2). Cephalometry revealed decreased SNB and, consequently, an increased ANB angle (Table 1). Superimposition of the pre- and post-treatment lateral cepholograms showed improvement of the mandibular prognathy, and normal inclinations of the maxillary and mandibular incisors (Fig. 3).

The postretention records indicated that a Class I molar and canine relationship was maintained, with good interdigitation, a symmetric facial and dental midline, and a good facial appearance (Fig. 4). All of the skeletal, dental and soft tissue measurements were preserved from 


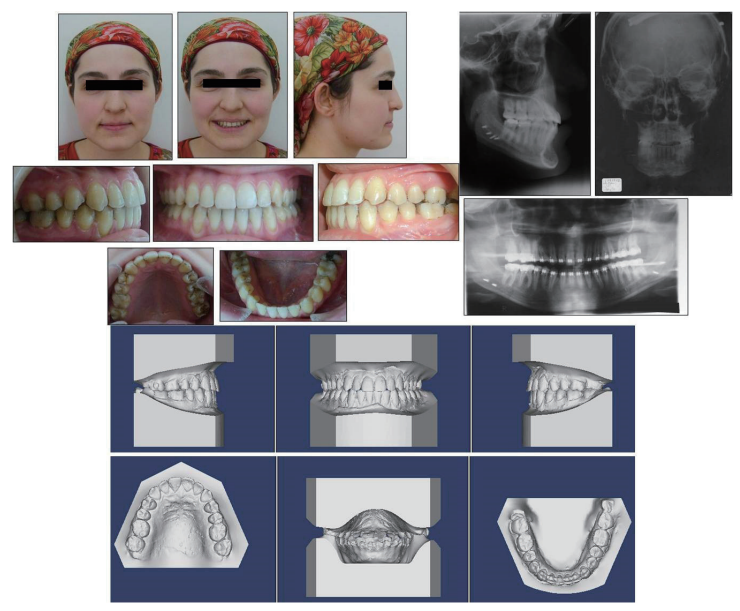

Fig. 2 Post-treatment facial and intraoral photographs, dental casts, lateral and posteroanterior radiographs, and panoramic radiograph just before removal of appliances.

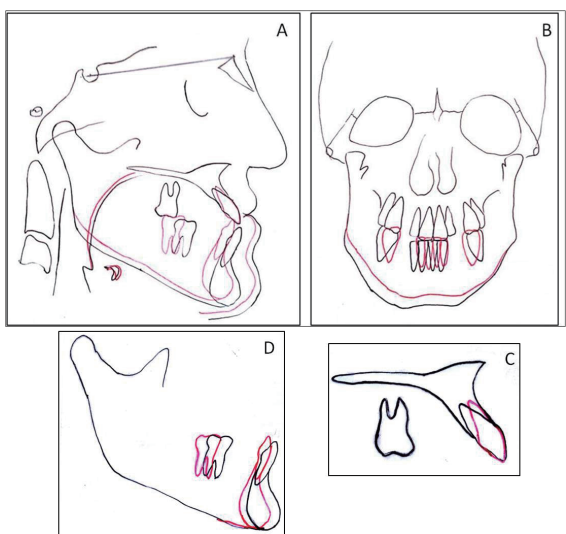

Fig. 3 Superimposed pretreatment (black line) and post-treatment (red line) lateral cephalometric and posteroanterior radiograph tracings: A, on the sella-nasion plane at the sella; $\mathrm{B}$, on the crista galli-ANS plane at the crista galli; $\mathrm{C}$, on the palatal plane at ANS; D, on the mandibular plane at the menton.

\section{T2 to T3 (Table 1, Fig. 5).}

The tongue space and hyoid bone position were evaluated in accordance with Kawakami et al. (6). The posterior vertical tongue space (D1) and horizontal (mesopharyngeal) tongue space (D2) showed a gradual decrease from $\mathrm{T} 1$ to $\mathrm{T} 3$, indicating that the tongue dorsum showed a posterior shift following surgery (Table 1, Figs. 3, 5). These decreases implied that the vertical and horizontal spaces around the tongue and the airway dimensions were reduced. The horizontal hyoid distance $(\mathrm{C} 3 \mathrm{H})$ increased from $\mathrm{T} 1$ to $\mathrm{T} 2$ and decreased from $\mathrm{T} 2$ to T3. The hyoid angle (NSH) was decreased from $\mathrm{T} 1$ to T2 and increased from T2 to T3 (Table 1, Figs. 3, 5), indicating that the hyoid bone was shifted anteriorly after surgery but returned toward its original position during the post-retention period.

The posteroanterior cephalogram showed that the right

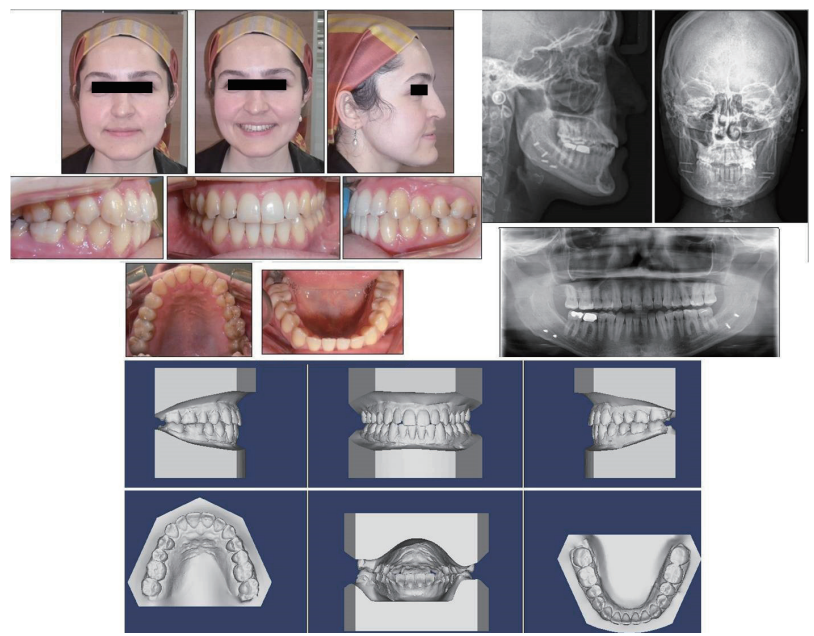

Fig. 4 Post-retention facial and intraoral photographs, dental casts, and cephalometric, posteroanterior and panoramic radiographs.

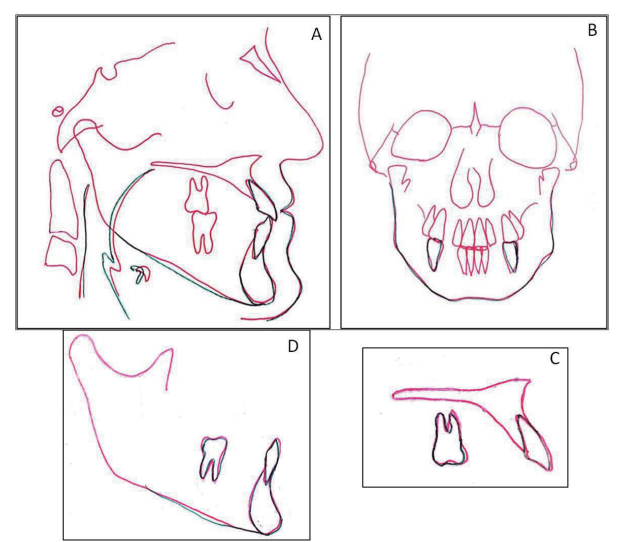

Fig. 5 Superimposed post-treatment (red line) and post-retention (green line) lateral cephalometric and posteroanterior radiograph tracings: A, on the sella-nasion plane at the sella; B, on the crista galliANS plane at the crista galli; $\mathrm{C}$, on the palatal plane at ANS; D, on the mandibular plane at the menton.

and left antegonion-midsagittal plane distance (AG-CsPl and $\mathrm{GA}-\mathrm{CsPl}$ ) measurements were matched by a $4-\mathrm{mm}$ horizontal movement of the right antegonion, indicating that the mandibular midline shift was eliminated (Table 1, Fig. 3). Eight years after the orthodontic treatment, the patient showed a stable skeletal and dental relationship (Table 1, Fig. 5).

\section{Discussion}

Skeletal Class III malocclusions are one of the most complicated dental deformities in both childhood and adulthood. The present case report demonstrates that orthognathic surgery can be effective for correction of skeletal Class III malocclusions and facial asymmetries.

A previous study of short- and long-term skeletal stability after mandibular setback surgery with BSSO has indicated that this procedure can be problematic, with 
relapse rates showing great variation from 1.0 to $91.3 \%$ (7). One of the main reasons for this tendency for relapse after orthognathic surgery is changes in the orofacial skeletal and soft tissue components, although tongue position and function may compensate for those changes in the oral environment. Kawakami et al. (6) stated that although they observed narrowing of the airway just after surgery, the vertical and horizontal spaces around the tongue were maintained at 1 month, the adaptive downward movement of the hyoid bone compensating for the airway reduction caused by mandibular setback. They also reported that more than 1 year after surgery, adaptation of the hyoid position returned, resulting in a significant decrease in airway dimension. Thus, mandibular setback causes airway narrowing in the late period after surgery, while the early postoperative airway dimension is maintained. Kawakami et al. (6) recommended that long-term evaluation should be performed because of the possibility of adaptive changes after mandibular setback surgery. In the present case, consistent with the previous study (6), although the posterior space around the tongue and airway dimensions were reduced and the hyoid position remained the same in the post-retention period, the patient still did not develop any breathing disorders.

Bicortical screw fixation was used in the present case. Roh et al. (7) have suggested that there are no significant differences in postoperative skeletal stability and condylar positioning after mandibular setback surgery using sliding plate, miniplate or bicortical screw fixation. In the present case, the bicortical screws were not removed at the patient's request after the healing period.

The results in the present case suggest that orthognathic treatment with bicortical screw fixation might be helpful for long-term correction of Class III malocclusion with facial asymmetry.

Proffit et al. (8) reported that bimaxillary surgery resulted in more stable outcomes, whereas mandibular setback procedures yielded less stable results when performed alone. The authors suggested that this was because mandibular growth continued for a longer period in mandibular prognathic patients than in individuals without this problem, and so would be more likely in the long term after surgery in patients who underwent mandibular setback surgery at a younger age. Profitt et al. (8) also reported that asymmetry with rigid fixation facilitated stabilized surgical movements. However, they noted that asymmetric movements in the mandible (rather than the maxilla) also tended to lead to relapse, and that chin relapse with more than $2 \mathrm{~mm}$ of movement occurred in $50 \%$ of patients.

In the present case, although mandibular sagittal split-ramus surgery was performed alone, with $3 \mathrm{~mm}$ of asymmetric movement, no tendency for relapse was observed in the clinical and radiological records. Several reasons could have accounted for this. For instance, the patient was 25 years old at the beginning of the treatment, and thus late growth would not have occurred in the sagittal plane or frontal plane within 8 years after treatment. Additionally, the use of rigid bicortical screw fixation may have prevented relapse.

The present case demonstrates the importance of longitudinal evaluation of dentofacial morphology and analysis of tongue and hyoid posture in skeletal Class III patients with mandibular asymmetry.

\section{Conflict of interest}

None of the authors had any conflict of interest regarding this case report.

\section{References}

1. Willmot DR (1981) Soft tissue profile changes following correction of class III malocclusions by mandibular surgery. Br J Orthod 8, 175-181.

2. Charles A, Subbiah S, Senkutvan RS (2014) Combined orthodontic and surgical management of a skeletal Class III malocclusion with mild asymmetry--a non extraction approach. Int J Dent Sci Res 2, 5-8.

3. Proffit WR, Phillips C, Dann C 4th (1990) Who seeks surgicalorthodontic treatment? Int J Adult Orthodon Orthognath Surg $5,153-160$

4. Nicodemo D, Pereira MD, Ferreira LM (2008) Self-esteem and depression in patients presenting angle Class III malocclusion submitted for orthognathic surgery. Med Oral Patol Oral Cir Bucal 13, E48-51.

5. Proffit WR, Phillips C, Turvey TA (1991) Stability after surgical-orthodontic corrective of skeletal Class III malocclusion. 3. Combined maxillary and mandibular procedures. Int J Adult Orthodon Orthognath Surg 6, 211-225.

6. Kawakami M, Yamamoto K, Fujimoto M, Ohgi K, Inoue M, Kirita T (2005) Changes in tongue and hyoid positions, and posterior airway space following mandibular setback surgery. J Craniomaxillofac Surg 33, 107-110.

7. Roh YC, Shin SH, Kim SS, Sandor GK, Kim YD (2014) Skeletal stability and condylar position related to fixation method following mandibular setback with bilateral sagittal split ramus osteotomy. J Craniomaxillofac Surg 42, 19581963.

8. Proffit WR, Turvey TA, Phillips C (2007) The hierarchy of stability and predictability in orthognathic surgery with rigid fixation: an update and extension. Head Face Med 3, 21. 